\title{
Nanostructured composite materials reinforced with nature-based nanocellulose
}

\author{
G. Vargas, J. Trifol, I. Algar, A. Arbelaiz, G. Mondragon, \\ S. C. M. Fernandes, F. Mujika \& A. Eceiza \\ University of the Basque Country, San Sebastian, Spain
}

\begin{abstract}
A better understanding of the relation between structure and function of cellulose-based hierarchical materials could provide the opportunity for developing new multi-functional composite materials and for designing nanostructured materials structurally optimised, using biodegradable renewable reinforcing materials. In this paper, recent advances by the authors' research group in the study of nanocomposites, reinforced with natural nanocellulose from both vegetal and bacterial sources, are presented. The extraction process of cellulose nanofibres and nanocrystals as well as the composite manufacturing process are detailed. Two composite systems are considered: one based on vegetal cellulose nanocrystals and another based on bacterial cellulose nanofibres. The presented results concern the morphology, thermal behaviour, mechanical properties and optical performance of those hierarchical nanocomposites.

Keywords: nanocomposites, nanocellulose, vegetal cellulose, bacterial cellulose.
\end{abstract}

\section{Introduction}

Some botanical structures have extraordinary mechanical behaviour combining high stiffness and high strength due to, among other reasons, the hierarchical arrangement of their structural parts: the ligno-cellulosic bundles. In general terms, those botanical structures are composed of semicrystalline cellulose-based sclerenchymatous stiff nanofibres bonded by a soft polymeric parenchymatous matrix. On that sense, cellulose nanofibres are the fundamental reinforcement units of cellulose-based materials and they are the strengthening phase of all subsequent structures (within trees, plants, some marine creatures and algae). 
Recently, some research work has been carried out [1, 2] considering nanocellulose-based building blocks for the development of new cellulose based multi-functional composites.

In addition, it is well known that cellulose is the most abundant organic substance in nature, and that it can be synthesized by terrestrial plants, some animals (e.g. tunicates), algae and a large number of microorganisms [3]. Cellulose consists of a chain of $\beta$ - $(1 \rightarrow 4)$-linked glucose residues, and due to its abundance, low cost and processing issues, it has attracted the attention of researchers on several application fields [4].

The main characteristics of the cellulose macromolecule are: renewable nature (a remarkable feature for addressing environmental issues), regular structure, high molecular weight and reactive hydroxyl groups. These properties have contributed to the use of cellulose as a potential reinforcement due to the combination of their high surface area, high aspect ratio and exceptional specific mechanical properties [5].

In this work, recent advances by the authors' research group in the study of polymeric nanocomposites, reinforced with natural nanocellulose from both vegetal and bacterial sources, are presented. In that sense, as in the case of hierarchical structuring of wood, where stiff cellulose fibres (offering structural properties) are joined by a softer hemicellulose and lignin matrix (providing toughness), in the studied nanocomposites the natural structure is explored: a bonding between stiff supporting fibres and soft polymer matrices.

\section{Vegetal cellulose}

Compared with animals, plants are very different anatomically: rather than producing blocks of skeletal material, each cell has its own exoskeleton. Such exoskeleton is the plant cell wall. This architecture originates a deep influence of cells arrangement on plant structural behaviour [6]. Thereby, vegetable sources (i.e. wood and non-woody plants) combine low density, good mechanical properties, low cost and biodegradability and offer multiple possibilities in dimensions, composition and morphology of fibrous structures that can be useful for the development of, among other applications, nanocellulosic fibre composites. Those structural characteristics are associated with the mimics of their hierarchically built anatomies developed and optimized in a long-term evolution process [7]. Such hierarchical structure spans nanoscale to macroscopic dimensions (fig. 1).

Cellulose nanofibres and nanocrystals (also called whiskers) can be extracted from the ligno-cellulosic fibres of plants by chemical, enzymatic and mechanical treatments, or combinations of them. Afterwards, plants and trees are considered an interesting renewable source of renewable nanostructured reinforcements.

In this work, to obtain nanocellulose from vegetal sources as reinforcement phase of polymeric nanocomposites, the sisal fibre has been considered as raw material. The composition of sisal fibres is: cellulose (50-74\%), lignin (8-11\%), hemicellulose $(10-14 \%)$, pectin $(1 \%)$ and wax $(2 \%)$. Due to its high cellulose content, high quantity cellulose nanofibres can be extracted from sisal fibres [8]. 
The extraction procedure of vegetal cellulose nano-reinforcements is displayed in fig. 2.

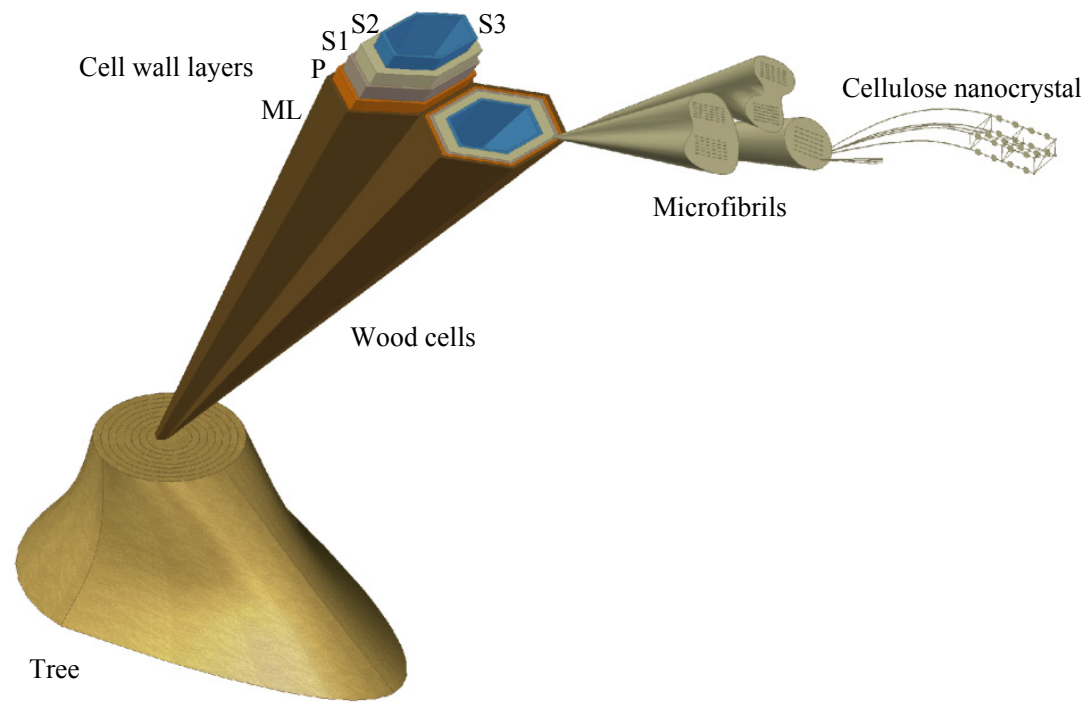

Figure 1: Scheme of a wood cell wall structure: middle lamellae (ML), primary wall (P), secondary walls (S1, S2, S3), microfibrils, crystalline structures.

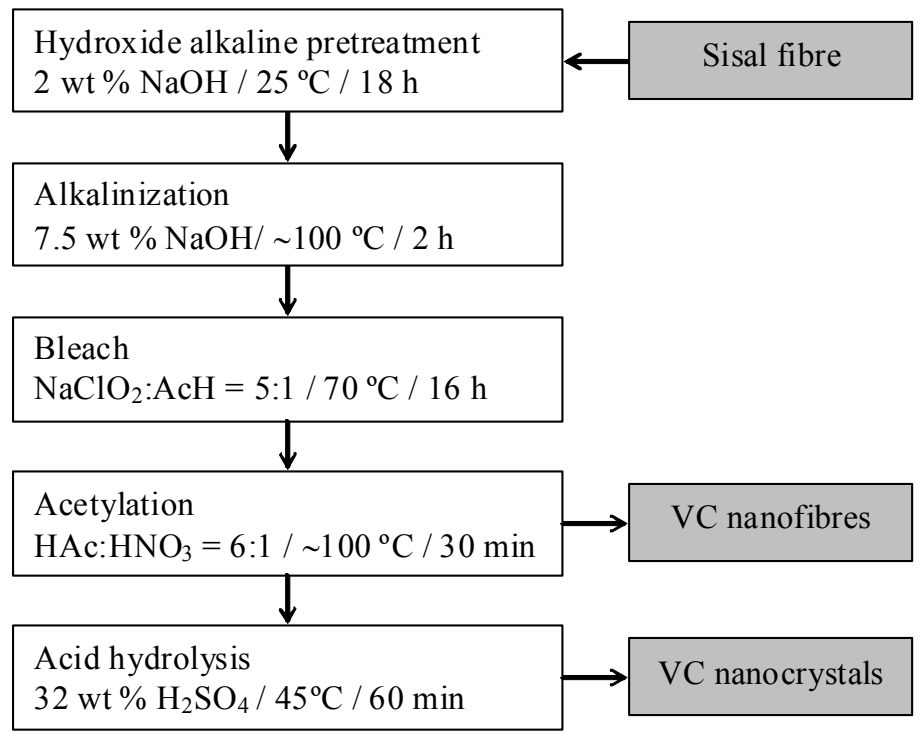

Figure 2: Scheme describing the procedure to extract nanocellulose from sisal fibre. 


\section{Bacterial cellulose}

Cellulose can also be biosynthesized by some bacteria, i.e. Gluconacetobacter xylinum, an aerobic gram-negative bacterium, that produces cellulose extracellularly as a primary metabolite from an organic source (e.g. glucose, fructose, sucrose, mannitol). The synthesis mechanism helps the aerobic bacteria to shift towards the oxygen-rich surface and a pellicle of nanocellulose is formed to both protect the cells from UV radiation, and retain moisture. Regarding chemical structure, bacterial cellulose (BC) is identical to vegetal cellulose (VC); nevertheless, $\mathrm{BC}$ is a three-dimensional micro- and nano-structure that presents higher crystallinity, water-holding capacity, mechanical strength, and high purity, because it does not contain lignin, hemicellulose, pectin or waxes, that are associated to wood and non-woody plants. Those issues make it interesting as raw material for several applications including composite reinforcement [3]. In fig. 3 , the production method of $\mathrm{BC}$ nano-reinforcements is presented [9].

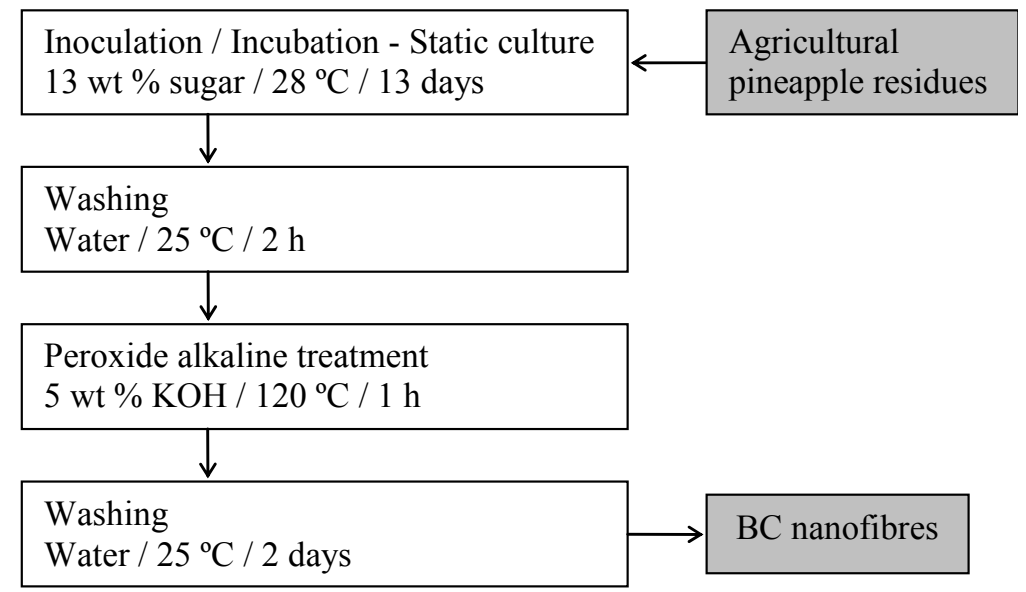

Figure 3: Scheme describing the procedure to extract bacterial cellulose nanocellulose.

\section{Nanocomposites based on nanocellulose}

Due to its outstanding mechanical and physical properties and its renewable/biodegradable character, cellulose is an excellent material to be incorporated as nanoreinforcement to polymers in order to achieve multifunctional composite materials. Two examples of composites reinforced with nature-based nanocellulose are detailed below. 


\subsection{PUR/VC nanocrystals}

Nanocomposites have been developed based on isocyanate-rich vegetal cellulose nanocrystals and their selective insertion in elastomeric polyurethane [5]. Nanoreinforcements have been functionalized by means of chemical reaction between the exposed hydroxyl functionalities on nanocrystals and isocyanate groups from an aliphatic diisocyanate, 1,6 -hexamethylene diisocyanate (HDI). After that, nanocrystals were incorporated to segmented thermoplastic polyurethane elastomers (STPUE), composed by alternating soft segments (SS) and hard segments (HS), by casting-evaporation method, obtaining composites with a reinforcement content of $1.5 \mathrm{wt} \%$.

The properties of the developed polyurethane nanocomposite have been evaluated by means of thermal, mechanical, and morphological characterization. Differential scanning calorimetry (DSC) scans were recorded on a Mettler Toledo $822 \mathrm{e}$ instrument, scanning samples from $-60^{\circ} \mathrm{C}$ to $150^{\circ} \mathrm{C}$ at $10^{\circ} \mathrm{C} / \mathrm{min}$ under nitrogen atmosphere. Dynamic mechanical analysis (DMA) was carried out in tensile mode (DMA Q-800, TA Instruments), applying a constant frequency of $1 \mathrm{~Hz}$, amplitude of $25 \mu \mathrm{m}$, a temperature range from $-90^{\circ} \mathrm{C}$ to $120^{\circ} \mathrm{C}$, a heating rate of $3^{\circ} \mathrm{C} / \mathrm{min}$ and a preload of $0.01 \mathrm{~N}$. Tensile tests were performed using an Insight 10 MTS universal machine with a load cell of $250 \mathrm{~N}$ at a crosshead speed of $100 \mathrm{~mm} / \mathrm{min}$, according to ASTM D 1708 standard [5].

\subsection{ESO/BC nanofibres}

A bionanocomposite of epoxidized soy-bean oil (ESO) matrix reinforced with bacterial cellulose nanofibres has been studied. Both constituents of the composite are obtained from renewable resources: ESO is manufactured by epoxidation of the double bonds of the SBO triglycerides with hydrogen peroxide and it is industrially available in large amounts at a reasonable cost, and $\mathrm{BC}$ nanofibres have been produced as explained on the above section. In addition, bacterial cellulose nanoreinforcement has been acetylated for enhancing both nanofibre dispersion and adhesion between constituents [10]. The preparation of $\mathrm{ESO} / \mathrm{BC}$ composite films with high $\mathrm{BC}$ content (up to $75 \mathrm{wt} \%$ ) has been carried out by the immersion manufacturing method, starting from BC film mats.

The experimental evaluation of optical, morphological and mechanical properties of nanocomposites was carried out. UV-visible transparency of composite films was characterized in a spectrometer Jasco UV-630. Tensile tests were carried out in a Minimat miniature mechanical tester machine with a $200 \mathrm{~N}$ load cell, considering a crosshead rate of $1 \mathrm{~mm} / \mathrm{min}$, and a distance between grips of $22 \mathrm{~mm}$, following ASTM D 1708 standard [10]. 


\section{Results and discussion}

\subsection{Vegetal cellulose (VC)}

In fig. 4, AFM phase images of vegetal cellulose nanocrystals surfaces are presented. Those images reveal that transverse dimension of VC nanocrystals are around $10 \mathrm{~nm}$, and that the length is $403 \pm 159 \mathrm{~nm}$, according to Mondragon et al. [11]. Besides, micrographs indicate that by means of the extraction procedure considered, the formation of nanoagglomerates is avoided. This situation contributes to subsequent adequate dispersion of $\mathrm{VC}$ nanoreinforcements on elastomeric polyurethane matrix, during the composite fabrication.

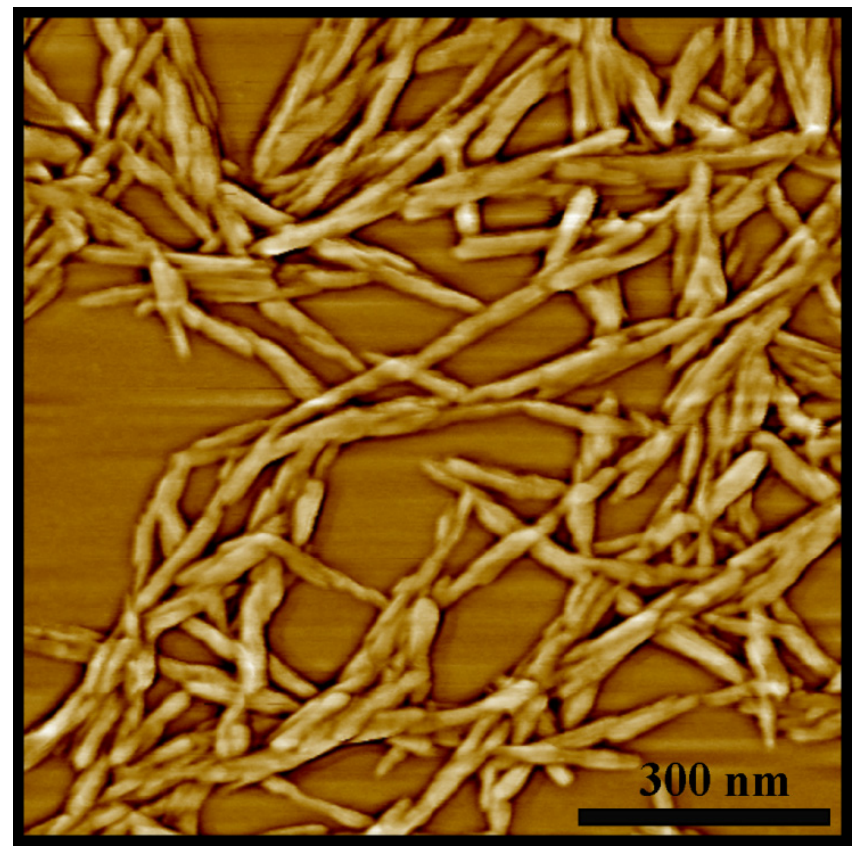

Figure 4: $\quad$ AFM phase image $(1 \mu \mathrm{m} \times 1 \mu \mathrm{m})$ of VC nanocrystals surface.

\subsection{Bacterial cellulose (BC)}

The morphological characterization of BC nanofibres by means of atomic force microscopy (AFM) reveals that the sample surface presents a highly fibrous network-like structure formed by bacterial ultrafine cellulose microfibrils, as exhibited in fig. 5. The average transverse dimension of $\mathrm{BC}$ nanofibres is 30 $35 \mathrm{~nm}$. 


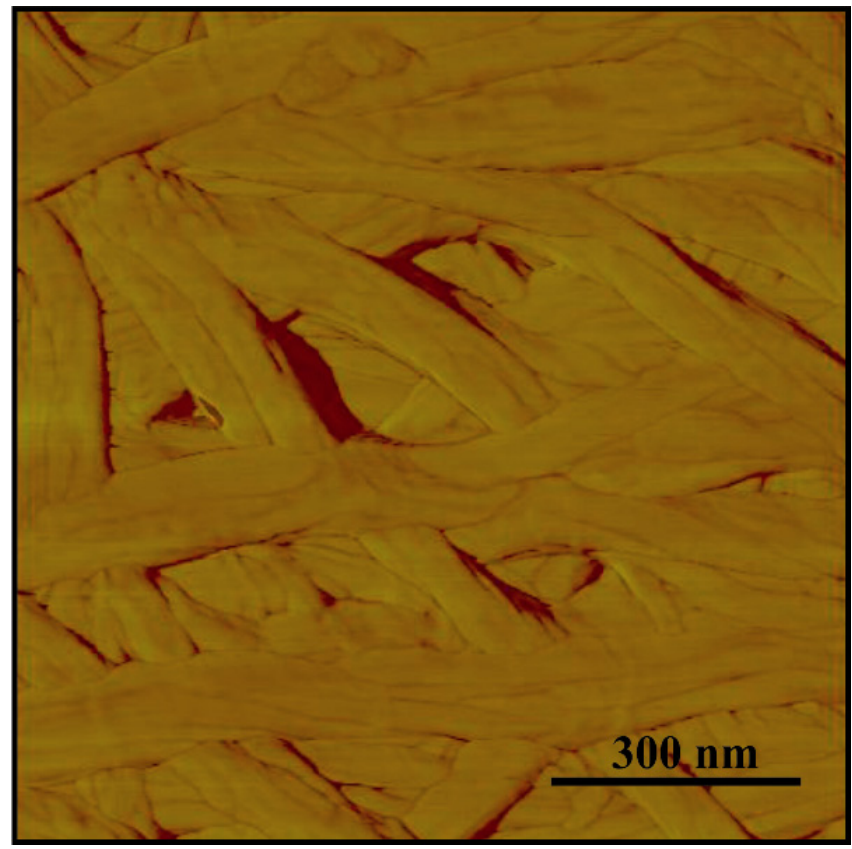

Figure 5: $\quad$ AFM phase image $(1 \mu \mathrm{m} \times 1 \mu \mathrm{m})$ of BC nanofibres surface.

\subsection{STPUE/VC nanocrystals}

The results for the thermal properties studied by DSC are presented in table 1, for neat polyurethane and its cellulose-based nanocomposites, both with functionalizated and non-functionalizated nanocrystals. Those results reveal that both STPUE matrix and VC nanocomposites exhibited the same temperature transitions and endotherms, i.e. melting temperature endotherm associated to soft segment $\left(T_{\mathrm{mSS}}\right)$, hard segment glass transition temperature $\left(T_{\mathrm{gHS}}\right)$, referred as enthalpy relaxation endotherm for polyurethanes with low hard segment content, and melting temperature endotherm associated to hard segment structures $\left(T_{\mathrm{mHs}}\right)$.

Table 1: $\quad$ DSC results for STPUE and their VC nanocomposites incorporating functionalizated and non-functionalizated nanocrystals [5].

\begin{tabular}{|l|l|l|l|l|}
\hline Sample & $T_{\mathrm{mSS}}\left({ }^{\circ} \mathrm{C}\right)$ & $T_{\mathrm{gHS}}\left({ }^{\circ} \mathrm{C}\right)$ & $T_{\mathrm{mHS}}\left({ }^{\circ} \mathrm{C}\right)$ & $\begin{array}{l}\Delta H_{\mathrm{mHS}} \\
(\mathrm{J} / \mathrm{g})\end{array}$ \\
\hline STPUE & -0.5 & 55 & 107 & 0.4 \\
\hline STPUE/VCnon-funct & -1 & 50 & 107 & 0.6 \\
\hline STPUE/VCfunct & -1 & 53 & 111 & 1.2 \\
\hline
\end{tabular}

DMA thermal results provide information about the viscoelastic properties of the studied materials, which can be related with microdomains thermal transitions. The typical behaviour of a segmented polymer, e.g. neat 
polyurethane, can be observed in three different zones: glassy state, first plateau, and second plateau. Comparing the DMA results of STPUE matrix with those of VC nanocomposites, favourable matrix-nanocrystal interactions can be observed, possibly due to the formation of a STPUE/VC interphase, which immobilize/reduce molecular mobility of matrix chains.

The tensile test results are summarized in table 2, considering the following properties: tensile modulus $(E)$, tensile strength at maximum elongation $(\sigma)$, yield strength at $50 \%$ offset $\left(\sigma_{50}\right)$, and elongation at break $\left(\varepsilon_{\mathrm{b}}\right)$. The STPUE/VC nanocomposites present a significant increase on $E$ and $\sigma_{50}$ compared to the unfilled polyurethane matrix. Composites with functionalizated nanocrystals exhibit an important decrease in $8 \mathrm{~b}$, which can be related to preferential interaction of HDI anchored chains with hard segment. It results in hard segments crystallization, offering good modulus and retaining a ductile behaviour [5].

Table 2: Tensile test results for neat polyurethane and the processed VC nanocomposites including functionalizated and non-functionalizated nanocrystals [5].

\begin{tabular}{|l|l|l|l|l|}
\hline Sample & $E(\mathrm{MPa})$ & $\sigma(\mathrm{MPa})$ & $\sigma_{50}(\mathrm{MPa})$ & $\varepsilon_{\mathrm{b}}(\%)$ \\
\hline STPUE & $9.2 \pm 0.4$ & $7.4 \pm 0.7$ & $1.8 \pm 0.2$ & $1529 \pm 257$ \\
\hline STPUE/VCnon-funct & $15.4 \pm 3.8$ & $9.4 \pm 1.3$ & $2.2 \pm 0.2$ & $1587 \pm 26$ \\
\hline STPUE/VCfunct & $21.0 \pm 4.4$ & $4.5 \pm 0.3$ & $2.3 \pm 0.3$ & $636 \pm 116$ \\
\hline
\end{tabular}

\section{$5.4 \mathrm{ESO} / \mathrm{BC}$ nanofibres}

In order to evaluate the transparency of both constituents (ESO, BC) and $\mathrm{ESO} / \mathrm{BC}$ nanocomposite, photographs have been taken and presented in fig. 6 . As can be seen, the neat cured ESO film is transparent, but the BC nanofibre film is mostly opaque white coloured. Moreover, the cured ESO/BC composite maintains the transparency of ESO matrix, indicating uniform dispersion of BC nanofibres, even at high $\mathrm{BC}$ contents (i.e. $75 \mathrm{wt} \%$ ).

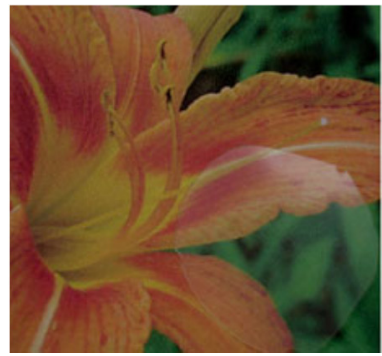

(a)

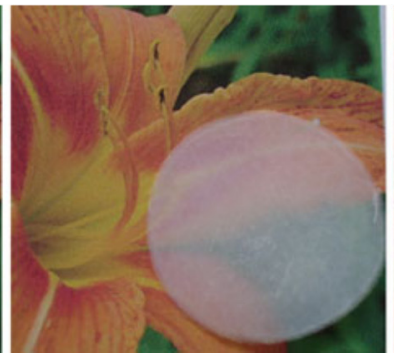

(b)

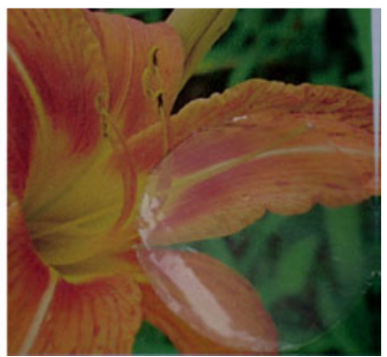

(c)

Figure 6: Transparency of studied films. (a) neat ESO matrix, (b) BC reinforcing mat, (c) ESO/BC composite. [10] with permission. 
$\mathrm{UV}$-vis tests results for the neat cured ESO matrix, $\mathrm{BC}$ mats and cured $\mathrm{ESO} / \mathrm{BC}$ composite films are displayed in fig. 7. The presented curves reveal that the cured ESO has a great degree of transparency throughout the analyzed wavenumber range. On the contrary, the $\mathrm{BC}$ films present a high degree of translucency. The cured ESO/BC composite film exhibits a high degree of transparency: compared with the cured ESO film it has only a $15 \%$ loss. Those UV-vis tests results are in agreement with images presented in fig. 6 .

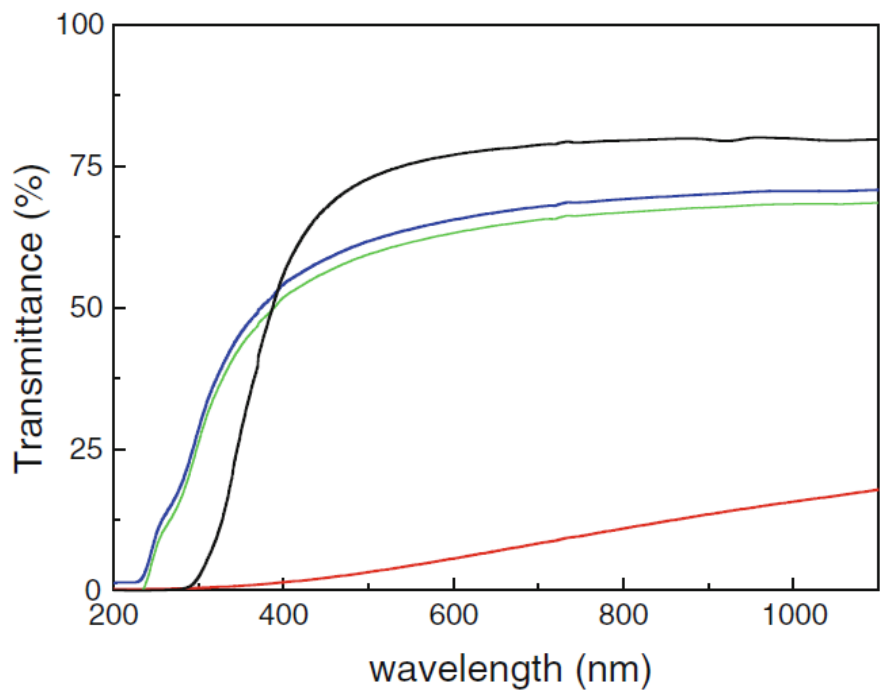

Figure 7: Light transmittance curves of studied films: (-) neat ESO matrix, (-) $\mathrm{BC}$ reinforcing mat, (-) ESO/BC composite 25 wt \%, (-) ESO/BC composite $75 \mathrm{wt} \%$. [10] with permission.

The mechanical results obtained by means of tensile tests are presented in table 3. As expected, in $\mathrm{ESO} / \mathrm{BC}$ composites as the $\mathrm{BC}$ nanofibre content increases, both stiffness and strength also increase, which is related to a uniformly distributed 3-D network of $\mathrm{BC}$ nanofibres in the composite. In that sense, ESO resin penetrates into the reinforcement mat voids, increasing the interaction between both constituents, preventing crack spread that would induce

Table 3: Tensile test results for neat ESO matrix, $\mathrm{BC}$ nanofibres film, and $\mathrm{ESO} / \mathrm{BC}$ composites.

\begin{tabular}{|l|c|c|c|}
\hline Sample & $E(\mathrm{MPa})$ & $\sigma(\mathrm{MPa})$ & $\varepsilon_{\mathrm{b}}(\%)$ \\
\hline ESO matrix & $450 \pm 100$ & $5.5 \pm 0.4$ & $3.6 \pm 0.5$ \\
\hline ESO/BC $25 \mathrm{wt} \%$ & $2,800 \pm 400$ & $25 \pm 0.4$ & $2.1 \pm 0.5$ \\
\hline ESO/BC $75 \mathrm{wt} \%$ & $5,900 \pm 500$ & $81 \pm 0.7$ & $2.1 \pm 0.4$ \\
\hline
\end{tabular}


catastrophic rupture. Moreover, the tensile elastic modulus of the $\mathrm{BC}$ composite exhibits a nearly linear relationship with the $\mathrm{BC}$ nanofibre content. Regarding ductility, the addition of $\mathrm{BC}$ nanofibres to ESO matrix decreases the composite strain at break. Nevertheless, considering the obtained tensile strength and maximum strain at break values, the $\mathrm{ESO} / \mathrm{BC}$ nanocomposites present a great capability for absorbing energy before breaking [10].

\section{Conclusions}

The development of both new biodegradable/renewable -based composite materials and nanostructured optimised materials depends, among others, on the understanding of the structure-to-function relation of such hierarchical systems. In that sense, this work presents current advances in the 'Materials + Technologies Group' of the University of the Basque Country related to the study of polymeric composites reinforced with both vegetal and bacterial nanocellulose. Two composite systems are considered: one based on a synthetic thermoplastic polyurethane elastomer (STPUE) matrix, and another based on renewable epoxidized soy-bean oil (ESO) matrix.

The isolation process of vegetal cellulose (VC) nanocrystals, and production method of bacterial cellulose (BC) nanofibres are mentioned, as well as the composite manufacturing process. Regarding vegetal cellulose, the results indicate that $\mathrm{VC}$ nanocrystals exhibit an aspect ratio between 55 and 25, and that agglomerates are avoided, which promotes adequate dispersion of nanocellulose on polymeric matrix. Considering bacterial cellulose nanofibres, morphological studies reveal a highly fibrous network-like structure formed by ultrafine cellulose microfibrils.

The thermal properties of neat polyurethane and STPUE/VC nanocomposites were investigated by differential scanning calorimetry (DSC) and dynamic mechanical analysis (DMA), and mechanical behaviour was studied by means of tensile tests. The results indicate that, in the case of thermal analysis, both STPUE matrix and VC nanocomposites exhibited the same temperature transitions and endotherms, and that there is a favourable matrix-nanocrystal interaction reducing molecular mobility of matrix chains. The mechanical performance of STPUE/VC nanocomposites is characterized by a reliable compromise between stiffness and ductility.

With regard to $\mathrm{ESO} / \mathrm{BC}$ nanocomposite, the results reveal that the obtained composite material presents high transparency and excellent mechanical properties, due to the hierarchical network-like structure of BC nanofibres, even at high bacterial cellulose content.

Those results lead to the consideration of polymeric composites reinforced with nanocellulose as important candidates for becoming hierarchical multifunctional materials in several industrial areas.

\section{Acknowledgements}

Technical and human support provided by Research Services (SGIker) of the University of Basque Country is gratefully acknowledged. Moreover, the authors 
wish to thank to the Centre for Industrial Technological Development (CDTI) of the Ministry of Economy and Competitiveness - Integrated Projects - for its financial support. Also, the authors would like to thank María Lozano for drawing Figure 1. This paper is dedicated In memoriam of Dr. Iñaki Mondragon Egaña.

\section{References}

[1] Moon, R.J., Martini, A., Nairn, J., Simonsen, J. \& Youngblood, J., Cellulose nanomaterials review: structure, properties and nanocomposites. Chemical Society Reviews, 40, pp. 3941-3994, 2011.

[2] Seppälä, J.V., Nanocellulose - a renewable polymer of bright future. Express Polymer Letters, 6(4), pp. 257-257, 2012.

[3] Castro, C., Zuluaga, R., Putaux, J.-L., Caro, G., Mondragon, I. \& Gañán, P., Structural characterization of bacterial cellulose produced by Gluconacetobacter swingsii sp. from Colombian agroindustrial wastes. Carbohydrate Polymers, 84(1), pp. 96-102, 2011.

[4] Zuluaga, R., Putaux, J.-L., Restrepo, A., Mondragon, I. \& Gañán, P., Cellulose microfibrils from banana farming residues: Isolation and characterization. Cellulose, 14(6), pp. 585-592, 2007.

[5] Rueda, L., Fernández d'Arlas, B., Zhou, Q., Berglund, L.A., Corcuera, M.A., Mondragon, I. \& Eceiza, A., Isocyanate-rich cellulose nanocrystals and their selective insertion in elastomeric polyurethane. Composites Science and Technology, 71, pp. 1953-1960, 2011.

[6] Ennos, A.R., Solid biomechanics, Princeton University Press: Princeton, 2012.

[7] Gañán, P., Zuluaga, R., Cruz, J., Vélez, J.M., Retegi, A. \& Mondragon, I., Elucidation of the fibrous structure of Musaceae maturate rachis. Cellulose, 15, pp. 131-139, 2008.

[8] Morán, J.I., Álvarez, V.A., Cyras, V.P. \& Vázquez, A., Extraction of cellulose and preparation of nanocellulose from sisal fibres. Cellulose, 15, pp. 149-159, 2008.

[9] Retegi, A., Gabilondo, N., Peña, C., Zuluaga, R., Castro, C., Gañán, P., de la Caba, K. \& Mondragon, I., Bacterial cellulose films with controlled microstructure-mechanical property relationships. Cellulose, 17, pp. 661669, 2010.

[10] Retegi, A., Algar, I., Martin, L., Altuna, F., Stefani, P., Zuluaga, R., Gañán, P. \& Mondragon, I., Sustainable optically transparent composites based on epoxidized soy-bean oil (ESO) matrix and high contents of bacterial cellulose (BC). Cellulose, 19, pp. 103-109, 2012.

[11] Mondragon, G., Fernandes, S., Retegi, A., Peña, C., Algar, I., Eceiza, A. \& Arbelaiz, A., A common strategy to extracting cellulose nanoentities from different plants. Industrial Crops and Products, 55, pp. 140-148, 2014. 